\title{
Steroid-Induced Dendritic Regression Reduces Anatomical Contacts between Neurons during Synaptic Weakening and the Developmental Loss of a Behavior
}

\author{
John R. Gray and Janis C. Weeks \\ Institute of Neuroscience, University of Oregon, Eugene, Oregon 97403-1254
}

\begin{abstract}
Steroid hormones alter dendritic architecture in many animals, but the exact relationship between dendritic anatomy, synaptic strength, and behavioral expression is typically unknown. In larvae of the moth Manduca sexta, the tip of each abdominal proleg (locomotory appendage) bears an array of mechanosensory hairs, each innervated by a planta hair sensory neuron (PH-SN). In the CNS, PH-SN axons make monosynaptic, excitatory nicotinic cholinergic connections with accessory planta retractor (APR) motoneurons. These synapses mediate a proleg withdrawal reflex behavior that is lost at pupation. The prepupal peak of ecdysteroids (molting hormones) triggers the regression of APR dendrites and a $>80 \%$ reduction in the amplitude of EPSPs produced in APRs by PH-SNs that innervate posterior planta hairs. The present study tested the hypothesis that a decrease in the number of synaptic contacts from PH-SNs to APRs contributes to this synaptic weakening. Pairs of PH-SNs and APRs were fluorescently labeled in larvae and pupae, and the number of indistinguishably close anatomical contacts (putative synapses) was counted by confocal laser scanning microscopy. During APR dendritic regression, the mean number of contacts from posterior PH-SNs decreased by $\sim 80 \%$, whereas the size of individual contacts did not change detectably and the axonal arbors of PH-SNs did not regress. These results suggest that the steroid-induced regression of motoneuron dendrites physically disconnects the motoneurons from the synaptic terminals of sensory neurons, producing synaptic weakening and the developmental loss of the proleg withdrawal reflex behavior at pupation.
\end{abstract}

Key words: steroid hormone; dendrite; synapse; Manduca sexta; development; confocal microscopy

\section{Introduction}

Steroid hormones can profoundly alter the size and complexity of dendritic arbors (for review, see García-Segura et al., 1994; Weeks and McEwen, 1997; McEwen, 2000). For example, in female rats, the rise and fall of estradiol during the estrous cycle regulates the cyclical increase and decrease in dendritic spine density on hippocampal pyramidal cells (for review, see Woolley, 1998). In male rats, androgen levels regulate the size of the dendritic arbors of motoneurons in the spinal nucleus of the bulbocavernosus (Kurz et al., 1986). Natural or experimentally induced changes in steroid hormone levels that alter dendritic architecture often simultaneously alter an animal's behavior; e.g., high estradiol levels at proestrous promote lordosis behavior in females, whereas circulating androgens support copulatory behavior in male rats. It is logical to assume that steroid-induced changes in dendritic arbors are accompanied by changes in synaptic connectivity (Matsumoto et al., 1988; Yankova et al., 2001) that contribute to changes in behavior. However, direct demonstration of this presumed chain of events in any one system is lacking.

Received July 24, 2002; revised Nov. 22, 2002; accepted Dec. 4, 2002.

This work was supported by National Institutes of Health Grant R01 NS23208 (J.C.W.). We thank Dr. Daniel Hartline for advice on three-dimensional reconstruction, Dr. David Lenzi for service as a blinded observer, and Dr. William M. Roberts, Dr. Johnathan Melville, M. JadeZee, and Ari Winbush for helpful suggestions on this manuscript.

Correspondence should be addressed to Janis C. Weeks, 1254 Institute of Neuroscience, University of Oregon, Eugene, 0R 97403-1254. E-mail: weeks@uoneuro.uoregon.edu.

J. R. Gray's present address: Department of Biology, University of Saskatchewan, 112 Science Place, Saskatoon, SK S7N 5E2 Canada.

Copyright $\odot 2003$ Society for Neuroscience $\quad 0270-6474 / 03 / 231406-10 \$ 15.00 / 0$
During metamorphosis of the moth Manduca sexta, steroid hormones [ecdysteroids, including 20-hydroxyecdysone (20E)] regulate dramatic changes in neurons and behavior (for review, see Levine and Weeks, 1989; Fahrbach and Weeks, 2002). A “prepupal peak" of $20 \mathrm{E}$ at the end of larval life triggers dendritic regression in motoneurons (see Fig. 1A) (Weeks et al., 1992), which are subsequently respecified for new functions (accompanied by dendritic regrowth) or eliminated by cell death during the pupal stage (Weeks and Truman, 1985; Weeks and ErnstUtzschneider, 1989; Weeks et al., 1992; Kent and Levine, 1993; Duch and Levine, 2000). Pupation also involves the loss of many larval behaviors, including the proleg withdrawal reflex (Weeks and Jacobs, 1987). This reflex is triggered by the deflection of the mechanosensory hairs located on the tip of each abdominal proleg (locomotory appendage) (see Fig. 1B) (Wiel and Weeks, 1996). Each hair is innervated by a single planta hair sensory neuron (PH-SN) (Peterson and Weeks, 1988), which makes monosynaptic, excitatory nicotinic cholinergic connections with the accessory planta retractor (APR) motoneuron and related motoneurons. The proleg withdrawal reflex is mediated by these direct sensorimotor synapses plus indirect interneuronal pathways (see Fig. 1C) (for review, see Weeks et al., 1997).

Streichert and Weeks (1995) showed that the mean amplitude of EPSPs evoked in APRs by PH-SNs decreases significantly (by $\sim 40-80 \%$, depending on PH-SN location) when APR dendrites regress (see Fig. $1 D$ ), although APR input resistance increases. They also provided indirect evidence that the size of PH-SN arbors does not change during this time. These findings suggested 


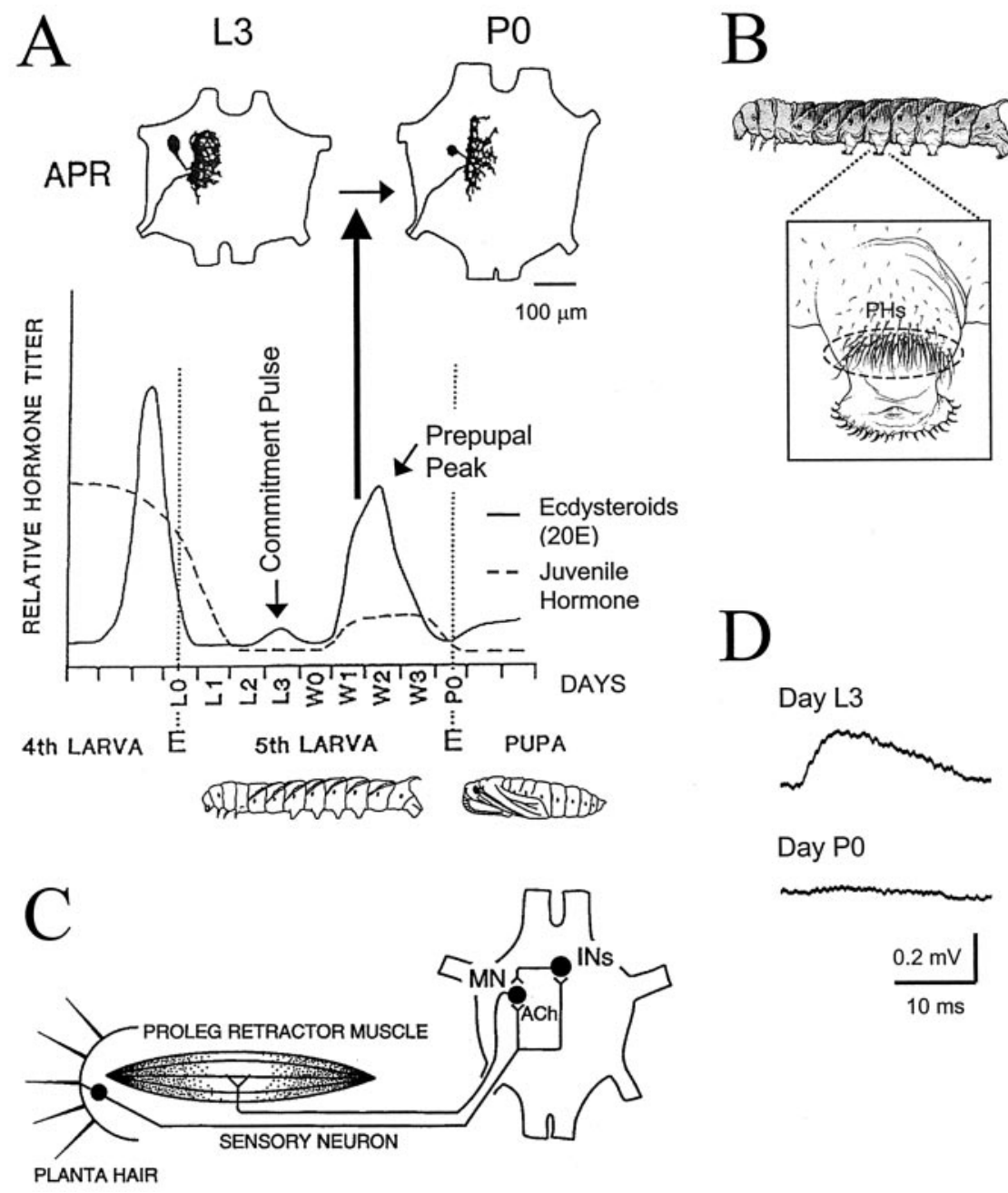

Figure 1. Steroid-mediated regression of APR dendrites and dismantling of the proleg withdrawal reflex during the larvalpupal transformation of Manduca. A, top, Camera lucida drawings show individual APRs stained with cobalt chloride on day L3 (left) and day PO (right). The outline of an abdominal ganglion is shown, with the anterior end up. The APR dendritic arbor is significantly reduced on day P0 (Streichert and Weeks, 1995; Sandstrom and Weeks, 1998). The timeline illustrates changes in relative hemolymph levels of ecdysteroids (solid line) and juvenile hormone (dashed line) from the late fourth larval instar to the early pupal stage [hormone titers redrawn from Bollenbacher et al. (1981) and Riddiford and Truman (1994)]. Developmental days relevant to this study are indicated along the horizontal axis. During the fifth (final) instar, the juvenile hormone titer drops and is followed by a small rise in ecdysteroids (20E) on day L3, termed the commitment pulse, and a larger rise in 20E spanning days $\mathrm{W} 1$ to $\mathrm{P0}$, termed the prepupal peak. The rise of the prepupal peak of $20 \mathrm{E}$ triggers dendritic regression in proleg motoneurons (bold vertical arrow) (Weeks and Truman, 1985, 1986; Weeks, 1987; Weeks et al., 1992). E, Ecdysis (shedding of the cuticle from the previous stage; indicated by vertical dotted lines); $L O$, day of ecdysis to the fifth larval instar; $L 1, L 2$, etc., days after $L 0 ; P O$, day of ecdysis to the pupal stage; $W 0$, day of wandering (when the larva ceases feeding and burrows underground); $W 1, W 2$, etc., days after wandering. $B$, Lateral view of a Manduca larva (anterior to the left) with the proleg in abdominal segment 4 enlarged (inset) to illustrate the dense array of PHs (enclosed by dashed oval) near the proleg tip. C, Neural circuit for the proleg withdrawal reflex in Manduca larvae. The proleg tip (left), a proleg retractor muscle (middle), and ganglion of the same abdominal segment (right; anterior is up) are shown schematically. Neurons are indicated by filled circles. Each PH is innervated by a single sensory neuron with a cell body in the proleg epidermis and an axon that projects to the ganglion. PH-SNs excite ipsilateral proleg retractor motoneurons $(M N)$, including the APR, via monosynaptic, nicotinic cholinergic (ACh) synapses and polysynaptic pathways through interneurons (INs) (reviewed by Weeks et al., 1997). The diagram shows the reflex circuit for the left proleg in a body segment; the circuit is duplicated for the right proleg (not shown). The ability of sensory input to evoke motor output via the circuit weakens dramatically during the larval-pupal transformation (Jacobs and Weeks, 1990; Streichert and Weeks, 1995). D, The size of EPSPs produced in APRs by PH-SNs decreases during the larval-pupal transformation. Traces show intracellular recordings from an APR on day L3 (top) and a different APR on day PO (bottom) while stimulating action potentials in a PH-SN located in the posterior region of the PH array to evoke monosynaptic EPSPs (signal-averaged from multiple trials; APR resting membrane potential set at $-60 \mathrm{mV}$ ). № EPSP was detectable on day PO. Data are from Streichert and Weeks (1995).

that decreased EPSP amplitude was associated with the physical disconnection of APR dendrites from the synaptic terminals of PH-SNs. The present study supported this hypothesis by demonstrating a developmental decrease in the number of close anatomical appositions between PH-SNs and APRs. These findings suggest that steroid-induced dendritic regression plays a key role in synaptic weakening and the elimination of a behavior during postembryonic life. Some results have been published previously in abstract form (Gray and Weeks, 1999).

\section{Materials and Methods}

Animals. M. sexta were reared individually on an artificial diet [modified from Bell and Joachim (1976)] under a 17/7 hr light/dark photoperiod and a $27 / 25^{\circ} \mathrm{C}$ temperature cycle. Insects were staged using standard developmental markers (see Fig. $1 A$ ). The day of ecdysis (cuticle shedding) to the fifth (final) larval instar is designated day L0, followed by days L1, L2, and L3. The onset of metamorphosis is marked by wandering behavior (day W0), when larvae cease feeding and seek a pupation site. The day of ecdysis to the pupal stage is designated day P0. All comparisons in this study were made between insects on day L3 and day P0.

Fluorescence labeling of sensory and motoneuron pairs. The $\mathrm{PH}$ array on each proleg tip is $\mathrm{di}-$ visible into anterior, middle, and posterior regions. The axon of each $\mathrm{PH}-\mathrm{SN}$ projects into the ganglion of the same abdominal segment, where the axon terminals form a somatotopic map based on the position of the $\mathrm{PH}$ in the array (Peterson and Weeks, 1988). The terminals of posterior $\mathrm{PH}-\mathrm{SNs}$ remain within the ganglion of the same body segment, whereas anterior and middle $\mathrm{PH}-\mathrm{SNs}$ send axons into neighboring ganglia and arborize there as well (Peterson and Weeks, 1988; Streichert and Weeks, 1995). We used posterior PH-SNs in the present study because the restriction of their terminals to a single ganglion facilitated complete staining of the entire axonal arbor (see below).

To stain an individual posterior PH-SN, insects were selected on day L2, anesthetized by chilling on ice for $45 \mathrm{~min}$, placed ventral side up in a Petri dish, and immobilized with strips of dental wax. The dish was filled with chilled water to a level just above the spiracles (respiratory openings) to maintain anesthesia. A well of petroleum jelly was formed around the tip of a proleg in abdominal segment 3 (A3) and filled with distilled water $\left(\mathrm{dH}_{2} \mathrm{O}\right)$. A single $\mathrm{PH}$ from the most ventral (distal), posterior region of the $\mathrm{PH}$ array was cut at its base using iridectomy scissors. After $10 \mathrm{~min}$, the $\mathrm{dH}_{2} \mathrm{O}$ in the well was replaced with a solution of $\sim 1 \%$ tetramethylrhodamine dextran (molecular weight, 3000; Molecular Probes, Eugene, OR; henceforth termed rhodamine) in $\mathrm{dH}_{2} \mathrm{O}$. The water covering the spiracles was removed, and the immobilized larva was held at $4^{\circ} \mathrm{C}$ for $36-48 \mathrm{hr}$ to reversibly arrest development and movement. The dye and petroleum jelly were then removed, and the larva was returned to standard rearing conditions to continue development.

Animals with a stained posterior $\mathrm{PH}-\mathrm{SN}$ were selected on either day L3 or day P0. Day L3 larvae were identified by their size and the presence of "frosted frass," signaling the occurrence of the commitment pulse of 20E (Fig. 1A) (Nijhout and Williams, 1974). Day P0 pupae were used for experiments within $1 \mathrm{hr}$ of completing ecdysis. 
Insects were sometimes held at $4^{\circ} \mathrm{C}$ for up to 24 $\mathrm{hr}$ to arrest development, before dissection. Animals were anesthetized on ice and opened dorsally under saline (see below), and ganglion A3 was removed with the lateral branch of the ventral nerve $\left(\mathrm{VN}_{\mathrm{L}}\right)$ left long on the side ipsilateral to the stained PH-SN. $\mathrm{VN}_{\mathrm{L}}$ carries the axons of the two APRs that innervate the ipsilateral retractor muscle and receive monosynaptic EPSPs from ipsilateral PH-SNs (Sandstrom and Weeks, 1996). Ganglia were placed ventral side up in a Sylgard-coated dish containing physiological saline composed of (in $\mathrm{mm}): 140 \mathrm{NaCl}, 5 \mathrm{KCl}, 4 \mathrm{CaCl}_{2}, 28$ glucose, and 5 HEPES, pH 7.4 (Trimmer and Weeks, 1989); they were then desheathed with fine forceps (Weeks and Jacobs, 1987) and observed under a Wild M3C dissecting microscope (Leica Microsystems, Bannockburn, IL) using substage dark-field illumination to visualize APR cell bodies.

The cell body of an APR ipsilateral to the stained $\mathrm{PH}-\mathrm{SN}$ was impaled with a borosilicate microelectrode filled at the tip with $4 \%$ Lucifer yellow $\mathrm{CH}$ (Molecular Probes) and backfilled with $0.5 \mathrm{M} \mathrm{LiCl}_{2}$ (electrode resistance, $\sim 30$ $\mathrm{M} \Omega$ ). APR was identified by its characteristic cell-body position and time-locked extracellular action potential recorded in the ipsilateral $V_{N_{L}}$ by means of a suction electrode led to a differential, alternating-current-coupled preamplifier (Sandstrom and Weeks, 1996). Lucifer yellow was iontophoresed into APR using 5-10 nA hyperpolarizing current pulses $(500 \mathrm{msec}, 1$ $\mathrm{Hz}$ ) generated by a Grass 88 stimulator (Grass Instruments, West Warwick, RI) connected to a Getting M5 intracellular amplifier (Getting Instruments, San Diego, CA). APRs were filled for at least $20 \mathrm{~min}$ or until action potentials were no longer detected. The electrode was withdrawn, $\mathrm{VN}_{\mathrm{L}}$ was crushed near the ganglion, and the preparation was left at room temperature for $30-45 \mathrm{~min}$ in the dark to permit dye diffusion. The ganglion was then fixed overnight in $4 \%$ paraformaldehyde at $4^{\circ} \mathrm{C}$, rinsed in PBS for 10 min, mounted on a poly-L-lysine-coated coverslip, dehydrated in an ascending series of ethyl alcohol, cleared in xylene, and mounted ventral side up in DPX mounting medium (Electron Microscopy Sciences, Fort Washington, PA). Slides were stored in the dark at room temperature until observed, usually within 2-4 d.

Confocal imaging. The quality of double fills was first assessed under epifluorescence using a Leitz (Wetzlar, Germany) Laborlux D compound microscope. If staining of the PH-SN and the APR appeared to be successful, the ganglion was then examined on a Zeiss (Oberkochen, Germany) LSM 310 confocal laser scanning microscope. Rhodamine fluorescence (red) was excited with a $568 \mathrm{~nm}$ laser line and detected through a $590 \mathrm{~nm}$ long-pass filter. Lucifer yellow fluorescence (green) was excited with a $488 \mathrm{~nm}$ laser line and detected through a $515-565 \mathrm{~nm}$ bandpass filter. To visualize anatomical contacts, ganglia were scanned using a $63 \times$ oil immersion objective [numerical aperture (NA), 1.25]; at this magnification, the entire $x, y$ extent of the $\mathrm{PH}-\mathrm{SN}$ arbor was contained within the field of view (see Fig. 4). Four scans of $1024 \times 1024$ pixels were averaged for each optical section, which were taken at $0.5 \mu \mathrm{m}$ intervals. This method produced images with a voxel resolution of $0.2 \times 0.2 \times 0.9$ $\mu \mathrm{m}$ in the $x$-, $y$-, and $z$-planes, respectively. For two-dimensional projections of optical sections, the total depth of tissue sampled was computed as $[($ number of intervals $\times 0.5 \mu \mathrm{m})+0.9 \mu \mathrm{m}]$. To ensure that scans in the two channels were in register, each optical section was scanned in the rhodamine D), $C, D, 30 \mu \mathrm{m}$.
L3

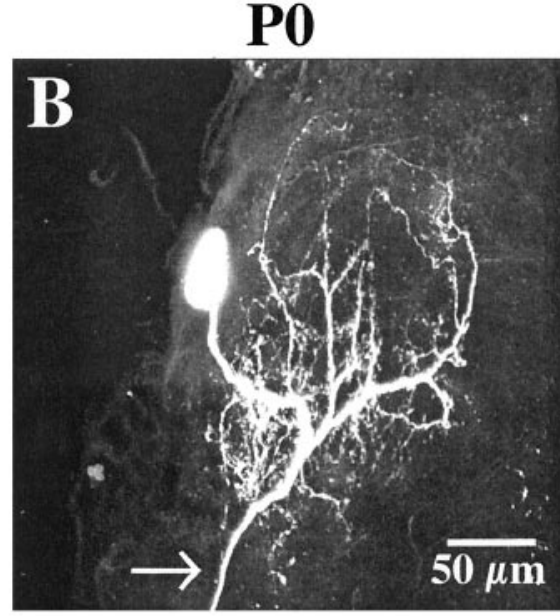

D
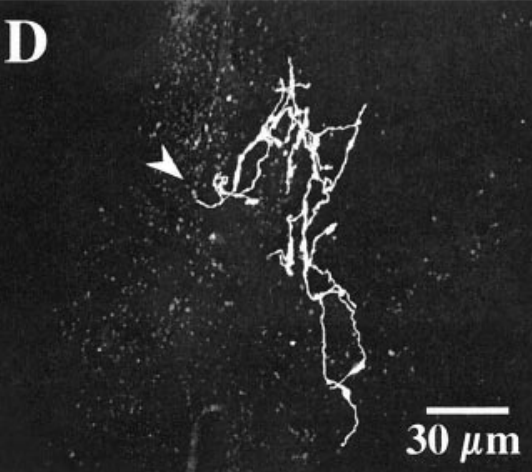

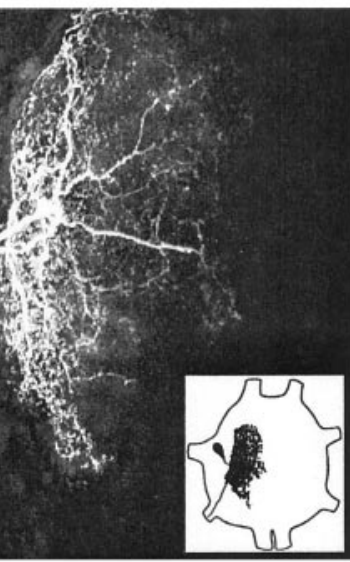

Figure 2. Morphology of APRs and posterior PH-SNs in larvae and pupae. Each panel is a two-dimensional projection of a stack a posterior PH-SN (C, D) on day L3 (left) or day PO (right). APRs were stained with Luciferyellow; PH-SNs were stained A) or a posterior PH-SN ( $O$ illustrated. $A, B$ The cell body of the APR appears at the left the dendritic arbor extends to the right, oward the ganglionic midline, and the axon projects laterally and posteriorly to exit the ganglion via a segmental nerve. The and posterior regions of ipsilateral neuropil. PH-SN arbors are similar in extent on days $L 3$ and PO. Scale bars: (in $B$ ) $A, B, 50 \mu \mathrm{m}$; (in

channel and the Lucifer yellow channel before moving to the next optical section. False color was used to identify the rhodamine (red) and Lucifer yellow (green) channels. The scanned volume contained the entire axonal arbor of the PH-SN and the portion of APR dendritic arbor that was coextensive with the PH-SN arbor. Accordingly, all potential sites of contact between a PH-SN and APR were imaged. A subset of putative synaptic contacts (see Fig. 5) was examined at higher magnification $(63 \times$ oil immersion objective and $3 \times$ hard magnification in the software).

The APR dendritic field spans the entire dorsoventral extent of the ganglion (Weeks and Jacobs, 1987) and extends more anteriorly than does the PH-SN arbor (see Fig. 7A). We did not image the entire APR dendritic arbor in double fills, because only the portion of the arbor that contacts PH-SNs was relevant to this study, and photobleaching of Lucifer yellow became a factor during the prolonged scanning required to visualize the entire APR arbor. In a few cases, the entire APR arbor was scanned using a $40 \times$ water immersion objective (NA, 1.2), which provided a larger field of view (see Fig. 2).

Identification of putative synaptic contacts. Only ganglia in which the arbors of both the PH-SN and the APR were completely and brightly stained in confocal images were used to count anatomical appositions. 
Staining was determined to be complete when adjacent segments of the processes of a neuron were continuous within each optical section and between adjacent sections, up to the point of termination of the process; i.e., there were no "gaps." Staining quality was also readily assessed by comparison with images of the same neurons stained with cobalt chloride (Weeks and Jacobs, 1987; Peterson and Weeks, 1988; Streichert and Weeks, 1995; Sandstrom and Weeks, 1996). Successful staining of APRs was routine, but only a low proportion of $\mathrm{PH}$-SNs were completely and brightly stained (Melville et al., 2003). Accordingly, the number of ganglia containing an acceptably stained pair of neurons was small ( $n=4$ on day L3; $n=3$ on day P0; see Results). Examination of less well stained material (data not shown) supported the conclusions based on ganglia used for quantitative analysis.

The series of optical sections from each ganglion was imported into Scion Image (Scion Corporation, Frederick, MD). The PH-SN (red) and APR (green) channels from each optical plane were combined into a single frame, and all frames from a ganglion were combined into a stack. Individual frames from each stack were displayed on a monitor and scored by eye for sites at which (1) pixels in the red and green channels overlapped (in which case the pixels appeared yellow) or (2) pixels in the red and green channels abutted with no discernible gap between (see Fig. 5) (Lamotte d'Incamps et al., 1998). We refer to sites of indistinguishably close anatomical contact in single frames as appositions. Three independent observers, who were kept unaware of the identity of the material, marked each apposition in each frame of each stack, without referring to adjacent frames. An apposition was included in the final data set when it was scored by at least two of the three observers; 90\% of appositions were identified by all three observers. A small number of appositions $(\sim 8 \%)$ were eliminated from the final data set when reconstruction of the PH-SN arbor (see below) revealed that these sites lay outside of the $\mathrm{PH}-\mathrm{SN}$ arbor, resulting from errant pixels in the rhodamine channel. Because of the narrow optical section thickness, appositions were often present in a similar $x, y$-location in consecutive frames. Counts of appositions were converted to counts of putative synaptic contacts (see Results) by eliminating duplicate counts of the same anatomical contact that was imaged in multiple consecutive frames. Thus, each putative synaptic contact was made up of one or more appositions scored in adjacent frames.

PH-SN morphometrics. The red channel was used to generate a stack of the PH-SN axonal arbor, which was reconstructed in three dimensions using a National Institutes of Health tree-tracing macro written by Dr. D. K. Hartline (University of Hawaii, Honolulu, HI; www.pbrc.hawaii. edu/ danh/Resources/treetracedoc.html). Total branch length and the number of branch points was measured for each reconstructed PH-SN. Reconstructions were then recombined with the $x$-, $y$-, and $z$-coordinates of the scored contacts to determine the distribution of putative synaptic contacts on the PH-SN arbor.

Statistical analysis. All statistical comparisons were performed using SigmaStat 2.0 statistical software (Jandel Scientific, San Rafael, CA). Differences were considered to be significant when $p<0.05$.

\section{Results}

\section{APR regresses while $\mathrm{PH}-\mathrm{SN}$ axonal arbors remain stable}

Previous quantitative studies demonstrated that the APR dendritic arbors in segments A3-A6 regress significantly between days L3 and P0 (Streichert and Weeks 1995; Sandstrom and Weeks, 1998); for these measurements, APRs were stained with cobalt chloride, the complete dendritic arbor was drawn as a two-dimensional projection, and the density of neuronal processes was calculated. In the present experiments, APRs were stained by the intracellular injection of Lucifer yellow. For technical reasons (see Materials and Methods), in ganglia that contained both a stained APR and a stained PH-SN, only the portion of the arbor of the APR that overlapped with PH-SN axon terminals was imaged. Therefore, we did not obtain quantitative measurements of the APR dendritic extent that could be compared directly with previous data. However, to verify that dendritic regression is apparent in Lucifer yellow-stained motoneurons, the complete arbor of the APR was imaged in ganglia that were not used to count appositions with PH-SNs. Similar to observations in cobalt-stained material (Weeks and Jacobs, 1987; Weeks and Ernst-Utzschneider, 1989; Streichert and Weeks, 1995; Sandstrom and Weeks, 1998), the neurites of APR were densely arrayed with fine, high-order processes that resembled dendritic spines (Fig. 2A,B). In other insect neurons, these processes typically bear input synapses (Peters et al., 1985) (see Discussion). The characteristic loss of dendrites between days L3 and P0 was readily apparent in Lucifer yellow-stained APRs (Fig. 2A,B); most major neurites (except those in posterior neuropil) were retained, whereas the density of smaller, higher-order branches decreased. Likewise, in ganglia used to count appositions, in which only the portion of the arbor of APR located within sensory neuropil was imaged, the dendritic extent of APR was markedly reduced on day P0 (see Figs. 4,7 ). These observations confirm that APRs undergo substantial dendritic loss between days L3 and P0 (Jacobs and Weeks, 1990; Streichert and Weeks, 1995; Sandstrom and Weeks, 1998).

Streichert and Weeks (1995) provided evidence that the axonal arbors of PH-SNs do not regress between days L3 and P0, but they did not quantify this observation specifically for the posterior PH-SNs used in the present study. Therefore, from three-dimensional reconstructions of individual rhodaminestained, posterior PH-SNs (Fig. 2C,D), we determined the mean number of branch points and total branch length of the central axonal arbors on days L3 and P0. Figure 3 shows that neither measure changed significantly during this period of development. As described previously in cobalt-stained material (Peterson and Weeks, 1988), the central axonal arbors of larval and pupal $\mathrm{PH}-\mathrm{SNs}$ stained with rhodamine were characterized by relatively smooth lengths of neurite punctuated by varicosities (Fig. 2C,D, see also Figs. 4, 5, 7). In other insect neurons, similar varicosities are presynaptic boutons (Peters et al., 1985). The lack
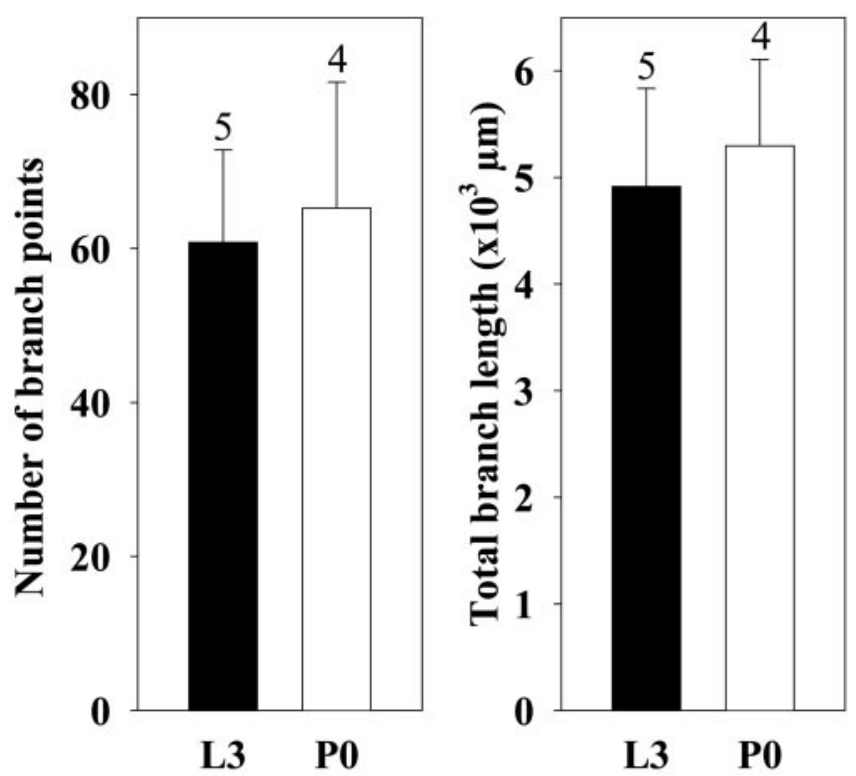

Figure 3. The central axonal arbors of posterior PH-SNs do not regress during the larvalpupal transformation. Columns indicate the mean number of branch points, and the total branch length, of the axonal arbors of posterior PH-SNs measured on day L3 (black columns; $n=$ 5 ) and on day PO (white columns; $n=4$ ). Error bars indicate SEM. The means on days $L 3$ and PO did not differ significantly (Student's $t$ test). 
of axonal regression in $\mathrm{PH}-\mathrm{SNs}$ is expected, given that these neurons are not respecified for new roles during metamorphosis (see Discussion).

These findings support the hypothesis that the axonal arbors of $\mathrm{PH}-\mathrm{SN}$ s remain stable between days L3 and P0 while the dendritic arbors of their postsynaptic targets, the APR motoneurons, regress.

\section{Identification of putative synaptic} contacts between APRs and PH-SNs We tested the hypothesis that the developmental weakening of the synapses from posterior PH-SNs to APRs is associated with a decrease in the number of synaptic contacts between the presynaptic and postsynaptic neurons. From each ganglion that contained a pair of completely and brightly stained neurons, we acquired a series of confocal optical sections at 0.5 $\mu \mathrm{m}$ intervals that spanned the entire volume of the PH-SN axonal arbor. Thus, all sites of potential anatomical contact between the PH-SN and APR were imaged. The depth of tissue sampled to image the complete PH-SN arbor was $38 \pm 4 \mu \mathrm{m}$ (mean \pm SEM) on day L3 $(n=4)$ and $50 \pm 8 \mu \mathrm{m}$ on day $\mathrm{P} 0(n=3)$.

In each optical plane, the images acquired in the red (PH-SN) and green (APR) channels were combined into a single frame. The frames were scored by multiple observers blind to the identity of the material for appositions in which pixels in the red channel overlapped with, or directly abutted, pixels in the green channel (see Materials and Methods). Some anatomical appositions appeared in multiple adjacent frames (Figs. 4, 5), so the counts of appositions were converted to counts of putative synaptic contacts by eliminating duplicate counts of the same anatomical apposition that appeared in more than one frame (see Materials and Methods). We use the term putative to refer to synaptic contacts because it is not possible to confirm the presence of active zones or functional synaptic transmission based on confocal microscopy (see Discussion). Similar methods have been used to identify putative synaptic contacts in other preparations (e.g., axo-axonic contacts of Ib fibers in cat spinal cord) (Lamotte d'Incamps et al., 1998).

Figure $4 A, B$ shows representative confocal images from days L3 and P0, depicting the same volume of sensory neuropil (3.4 $\mu \mathrm{m}$ depth) at both developmental stages. Arrows mark putative synaptic contacts between the axonal arbor of a posterior PH-SN and the APR dendritic arbor. More putative synaptic contacts were apparent on day L3 than on day P0 (see quantitative comparisons below). Putative synaptic contacts between $\mathrm{PH}-\mathrm{SN}$ and APRs are shown at higher magnification in Figure 5. Figure $5 A$ shows seven consecutive frames spanning the contact marked by an asterisk in Figure $4 A$; appositions were scored in four consecutive frames, indicated by overlap of the red and green channels.
Figure $5 B$ shows a single frame from a different preparation illustrating a PH-SN varicosity (red) and an APR dendrite (green) that abutted with negligible overlap.

The number of putative synaptic contacts decreases during the larval-pupal transformation, but contact size does not change

Figure $6 A$ compares the number of putative synaptic contacts between posterior PH-SNs and APRs on days L3 and P0. The mean number of putative contacts decreased significantly from 42 to 9 over this period of development, representing a $79 \%$ decrease. Concomitantly, the mean amplitude of EPSPs produced in APRs by posterior PH-SNs is $\sim 0.30 \mathrm{mV}$ on day L3 and $\sim 0.05 \mathrm{mV}$ on day P0 (Fig. 1D) (Streichert and Weeks, 1995) (see Discussion).

Synaptic strength can be influenced by both the number of contacts between presynaptic and postsynaptic neurons and the size of each synaptic contact; larger contacts may contain more active zones 

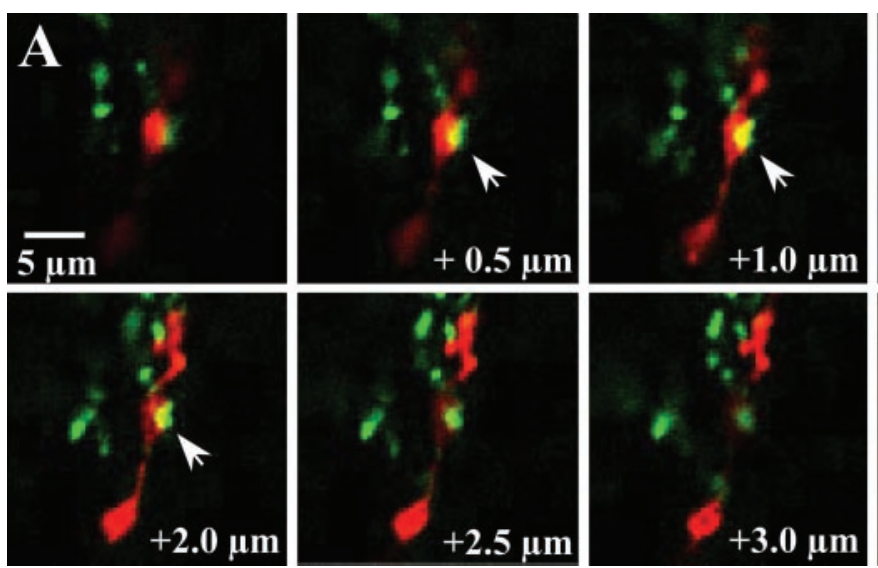

Figure 5. Putative synaptic contacts between posterior PH-SNs and APRs viewed at higher magnification. A, Seven sequential frames that spanned the volume of neuropil containing the putative synaptic contact marked with an asterisk in Figure 4. Numbers at the bottom right of each panel indicate the depth of the section compared with the first image in the series. Overlapping appositions (arrows) between the PH-SN (red) and APR ( green), indicated by yellow pixels, occurred in four consecutive frames, indicating that this putative synaptic contact spanned a depth of $\sim 2.9 \mu \mathrm{m}$. B, One frame from a different preparation on day $\mathrm{L} 3$ in which the processes of a posterior PH-SN and an APR abutted with negligible overlap (arrow). The scale bar refers to all panels.

and release more neurotransmitter (Grantyn et al., 1984). Having demonstrated a developmental decrease in the number of putative synaptic contacts between PH-SNs and APRs (Fig. 6A), we tested whether the size of the contacts changed. Optical section thickness was constant $(0.5 \mu \mathrm{m})$, so the number of adjacent frames in which a putative synaptic contact appeared provided a rough estimate of the size of the contact. The number of adjacent frames in which individual contacts appeared ranged from 1 to 7 , with a mean of $1.74 \pm 0.12$ (SEM) frames on day L3 $(n=167)$ and $1.90 \pm 0.30$ frames on day P0 $(n=28)$. These values did not differ significantly (Student's $t$ test). Conversion of these figures to physical dimensions provides an estimate of the mean size of putative synaptic contacts (measured along the $z$-axis) of 1.25-1.35 $\mu \mathrm{m}$ (range, $0.5-4.4 \mu \mathrm{m}$ ). These values are similar to the sizes of contacts observed in the $x, y$-plane (Fig. 5) and appropriate for insect central synapses (see Discussion).

We also compared the proportion of contacts that fell into different size categories at the two developmental stages. Figure $6 \mathrm{~B}$ shows that the distribution of contact size was similar on days L3 and P0; most contacts appeared in only one or two adjacent frames, and $>90 \%$ of the contacts appeared in three or fewer frames. The proportion of putative synaptic contacts in each size category did not differ significantly on days L3 and P0 (Fig. 6B). Thus, the number of putative synaptic contacts between PH-SNs and APRs decreased between days L3 and P0, but the size of the contacts did not change detectably.

We did not image the complete APR dendritic arbor in ganglia containing a stained $\mathrm{PH}-\mathrm{SN}$ (see above), so we did not reconstruct the three-dimensional structure of the motoneuron and determine the spatial distribution of putative synaptic contacts from PH-SNs. Qualitatively, most putative synaptic contacts from posterior $\mathrm{PH}-\mathrm{SN}$ occurred on the high-order branches of APR and not on large neuritic trunks (Figs. 4, 5). However, because we obtained a complete image of each PH-SN arbor, it was possible to reconstruct the sensory neuron arbors in three dimensions and to map the locations of putative synaptic contacts with APR. Figure 7A shows representative reconstructions of posterior $\mathrm{PH}-\mathrm{SNs}$, displayed as two-dimensional projections, with the portion of the APR dendritic arbor that occupied the same volume of neuropil superimposed. The size of the APR dendritic arbor that was coextensive with the $\mathrm{PH}-\mathrm{SN}$ axonal arbor was substantially
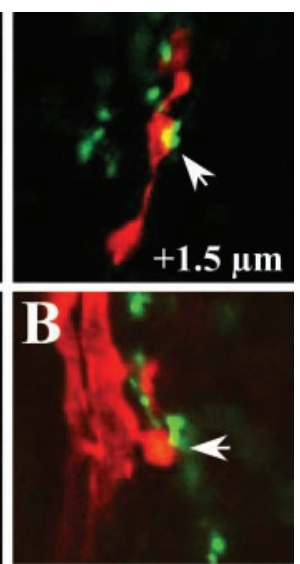

reduced on day $\mathrm{P} 0$. Figure $7 B$ shows the same $\mathrm{PH}-\mathrm{SN}$ reconstructions as in Figure $7 A$, with putative synaptic contacts with APR marked. As documented above (Fig. 3 ), the size of PH-SN axonal arbors appeared similar on days L3 and P0. In this example, there were 43 putative synaptic contacts from the PH-SN to APR on day L3 and four putative synaptic contacts from the PH-SN to APR on day P0. The loss of APR dendrites in posterior neuropil (Fig. $2 B$ ) was reflected in the dearth of putative synaptic contacts in the posterior region of the $\mathrm{PH}-\mathrm{SN}$ arbor on day $\mathrm{P} 0$.

\section{Discussion}

Previous studies demonstrated that the steroid-induced regression of the dendritic arbor of motoneuron APR is associated with a decrease in the amplitude of monosynaptic EPSPs from PH-SNs and the developmental loss of a proleg withdrawal reflex (Jacobs and Weeks, 1990; Streichert and Weeks, 1995). The present study used double fluorescence confocal microscopy to test the hypothesis that physical disconnection of APR dendrites from the synaptic terminals of $\mathrm{PH}-\mathrm{SNs}$ contributes to the synaptic weakening and behavioral loss. The dendritic morphology of APRs stained with Lucifer yellow on days L3 and P0 (Figs. 2, 4) was indistinguishable from that of APRs stained with cobalt at these stages (Fig. 1A) (Streichert and Weeks, 1995; Sandstrom and Weeks, 1998). In particular, the region of APR dendritic arbor that overlaps with $\mathrm{PH}-\mathrm{SN}$ axon terminals was regressed (Fig. 7A) (Jacobs and Weeks, 1990). The present study (Fig. 3; also see Figs. 2C,D, 7B) improved on previous indirect evidence that $\mathrm{PH}-\mathrm{SN}$ axonal arbors do not regress at pupation (Streichert and Weeks, 1995). Thus, fluorescently stained material viewed by confocal microscopy corroborated previous observations that $\mathrm{PH}-\mathrm{SN}$ arbors are structurally stable while the dendrites of proleg motoneurons regress at pupation.

Staining APRs with Lucifer yellow was routine, whereas staining $\mathrm{PH}-\mathrm{SN}$ s with rhodamine via the hair shaft had a low success rate that limited the number of double fills in which contacts could be counted. Only brightly and completely stained neurons were used for quantitative analysis (see Materials and Methods). The mean number of putative synaptic contacts between a posterior PH-SN and an APR decreased from 43 on day $\mathrm{L} 3$ to nine on day P0 (Fig. 6A), whereas the size of individual contacts did not change detectably (Fig. 6 B). The $79 \%$ decrease in the number of putative synaptic contacts parallels an $83 \%$ decrease in the mean amplitude of the EPSPs at these synapses (Streichert and Weeks, 1995). Because the input resistance of APR increases by $40 \%$ during this period (Streichert and Weeks, 1995), the actual decrease in synaptic current may exceed $83 \%$. Furthermore, the loss of posterior dendrites in APR (Figs. $1 A, 2 B, 7 A$ ) should preferentially eliminate $\mathrm{PH}-\mathrm{SN}$ inputs that are electrotonically the most distant from the cell body of the APR (Fig. 7) and that make a lesser contribution to EPSP amplitude. Nevertheless, EPSP amplitude is severely reduced on day P0. The correspondence between the developmental decrease in the number of putative synaptic contacts and EPSP amplitude supports the idea that these two features are related.

For technical reasons (see Materials and Methods) we used 
A
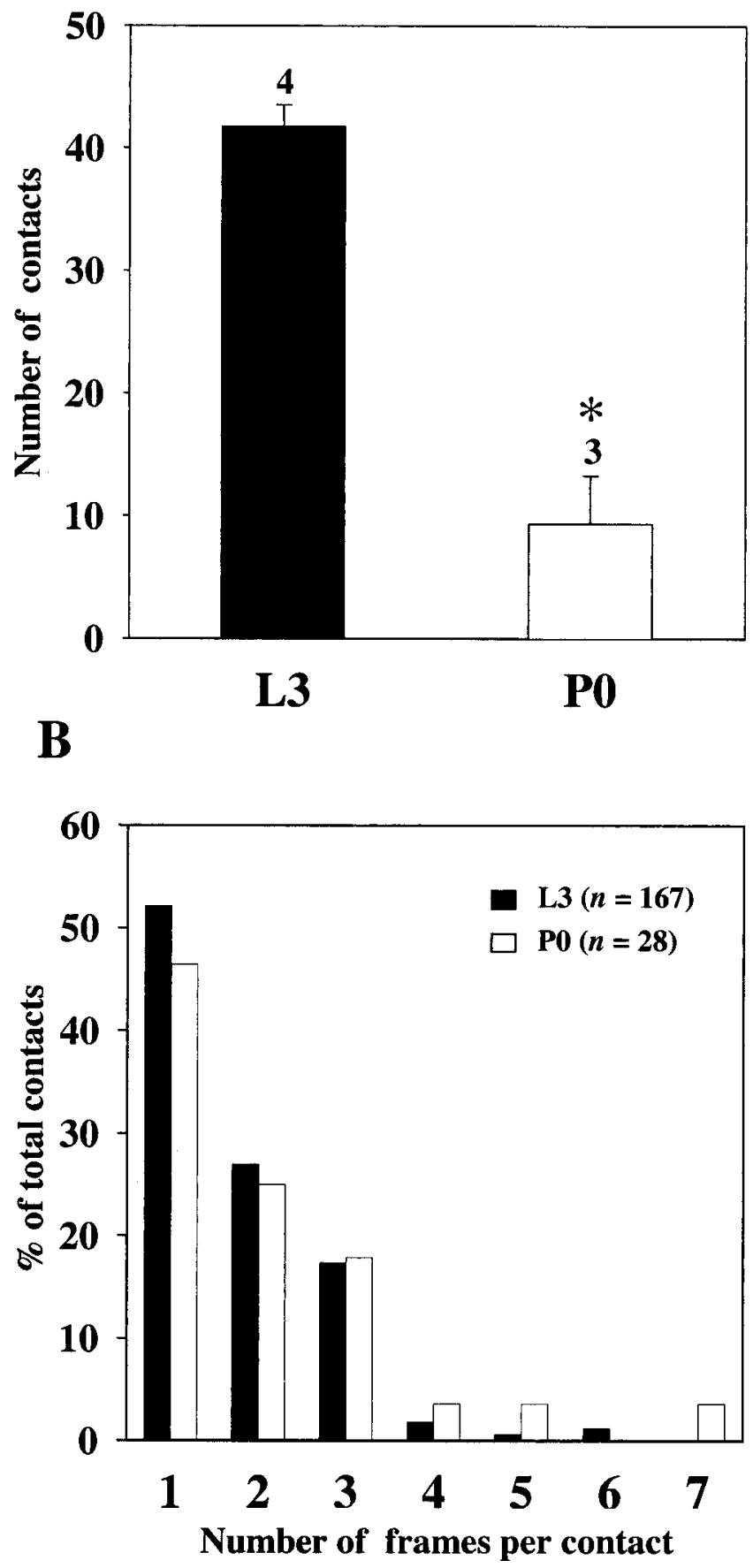

Figure 6. Quantification of the number and size of putative synaptic contacts between posterior PH-SNs and APRs on days $\mathrm{L} 3$ and P0. A, Columns indicate the mean number of putative synaptic contacts between posterior PH-SNs and APRs on day L3 (black column; $n=4$ ) and on day PO (white column; $n=3$ ). Error bars indicate SEM. The number of putative synaptic contacts was significantly reduced on day P0. ${ }^{*} p<0.001$; Student's $t$ test. $B$, The putative synaptic contacts identified in all preparations on day L3 ( $n=167$ contacts) and on day PO $(n=28$ contacts) were divided into categories based on the number of adjacent frames (at $0.5 \mu \mathrm{m}$ intervals) in which the contact appeared (range, 1-7 frames). The proportion of contacts in each category is expressed as a percentage of the total number of contacts counted at that stage. There were no significant differences in the proportion of contacts in each size category (i.e., contacts observed in one frame, two frames, etc.) on days $\mathrm{L} 3$ and $\mathrm{PO}\left(\chi^{2}\right.$ analysis). only posterior PH-SNs. However, because EPSP amplitude decreases significantly for $\mathrm{PH}-\mathrm{SNs}$ located in all regions of the array (Streichert and Weeks, 1995), we expect that the contact number likewise decreases for all $\mathrm{PH}-\mathrm{SNs}$.

\section{Validity of the analysis}

These results raise two major issues of interpretation. The first concerns the identification of indistinguishably close contacts observed at the light microscopic level as synapses, and the second concerns synaptic strength. Several lines of evidence support the designation of appositions between PH-SNs and APRs as putative synapses. First, the size and shape of the contacts resemble central synapses in other systems. Putative synapses were identified as indistinguishably close or overlapping profiles in images with a pixel resolution of $0.2 \mu \mathrm{m}$, with the mean contact length in the $z$-axis estimated to be $1.25-1.35 \mu \mathrm{m}$ (see Results). This size is consistent with other insect sensorimotor synapses (e.g., output synapses from locust wing hinge stretch receptor neurons to wing motoneurons) (Peters et al., 1985) and central synapses in general (for review, see Edwards, 1995). Most contacts occurred en passant from $\mathrm{PH}-\mathrm{SN}$ varicosities onto highorder dendrites of an APR (Fig. 5). Axonal varicosities (boutons) typically contain output synapses in insects [cockroach (Blagburn et al., 1985), locust (Watson and Burrows, 1985), Manduca (Sun et al., 1997)] and other species (DeRiemer and Macagno, 1981; Glanzman et al., 1989; Tse et al., 1991; Magoski and Bulloch, 1997). Output synapses may also be made by fine, smooth processes (Watson and Burrows, 1982; Granzow et al., 1985; Walmsley et al., 1987; Lamotte d'Incamps et al., 1998). Close appositions observed at the light microscopic level have been confirmed to be synapses by electron microscopy in a number of cases (Muller and McMahan, 1976; Pilowsky et al., 1990; Sorra and Harris, 1993; Buhl et al., 1994; Gonchar et al., 2002). In summary, the morphology of appositions between PH-SNs and APRs is entirely consistent with synaptic contacts, an assumption that can be tested in future experiments.

A second key issue in interpreting our results is the strength of individual synaptic contacts between PH-SNs and APRs. The amplitude of an EPSP reflects the number of release sites and the size of the synaptic current produced at each release site, which is in turn determined by the amount of neurotransmitter released, the number of receptors, and other factors (for review, see Edwards, 1995). The size of contacts between PH-SNs and APRs did not change detectably between days L3 and P0 (Fig. 6B), but more subtle alterations in synapse structure that could affect transmission (e.g., a decrease in the number of active zones or in the size of the postsynaptic density) are undetectable at the light microscopic level. It is well established that synaptic strength and synaptic morphology can be regulated independently (Tsujimoto et al., 1990; Stewart et al., 1996; for review, see Davis and Goodman, 1998), so the developmental weakening of synapses from PH-SNs to APRs could be accomplished entirely by electrophysiological changes rather than by altering the number of contacts. However, analysis of EPSP time-to-peak and the intrinsic electrical properties of APRs suggest that the developmental decrease in EPSP amplitude results from a decrease in binomial $n$ (i.e., the number of contacts or release sites) rather than from other factors (Streichert and Weeks, 1995). Evidence against the possibility that a change in synaptic release properties from $\mathrm{PH}-\mathrm{SN}$ s is involved in synaptic weakening comes from heterochronic mosaic animals, in which $\mathrm{PH}-\mathrm{SN}$ are maintained in the larval state by treating their somata with juvenile hormone, whereas APRs undergo nor- 

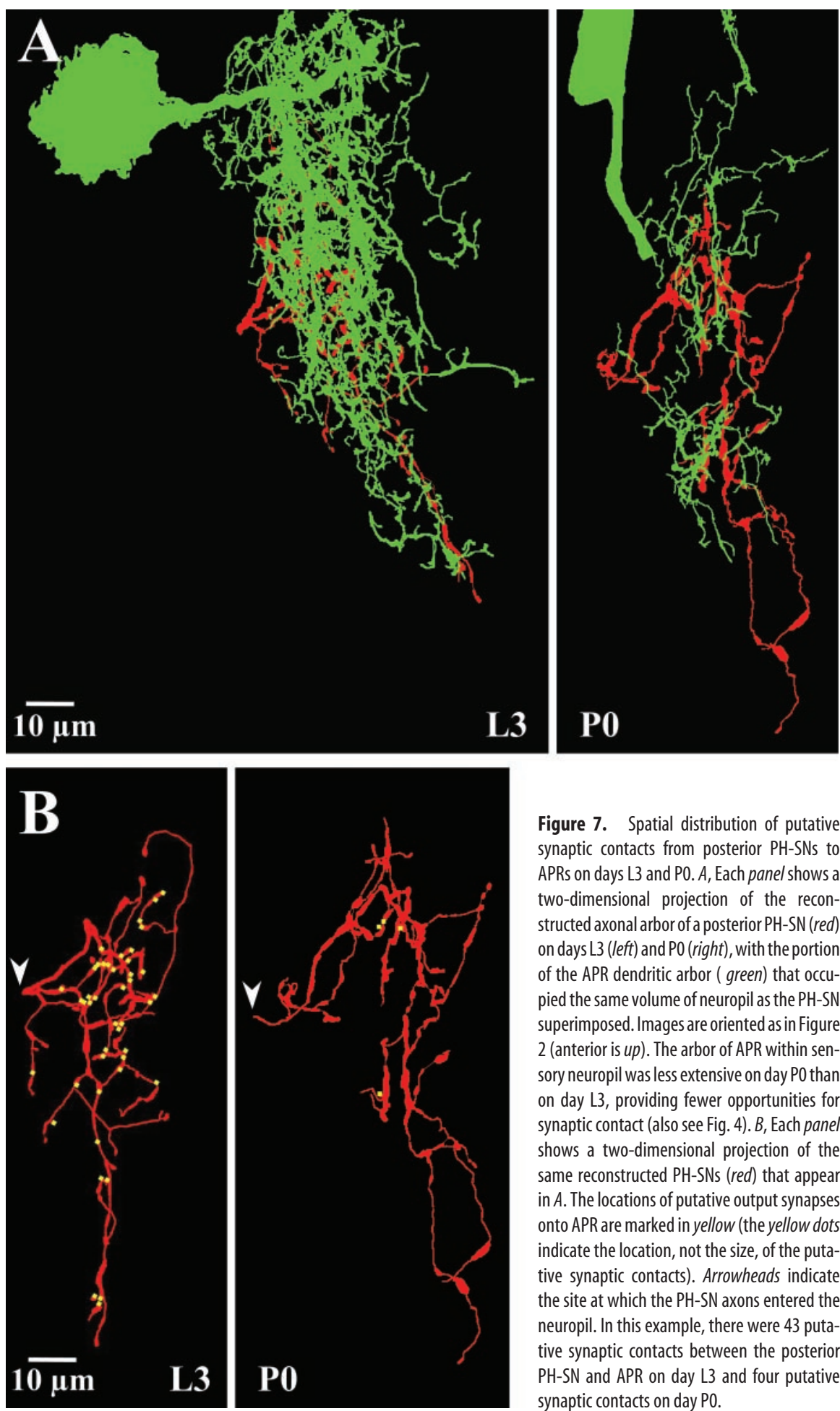

Figure 7. Spatial distribution of putative synaptic contacts from posterior PH-SNs to APRs on days $L 3$ and PO. A, Each panel shows a two-dimensional projection of the reconstructed axonal arbor of a posterior PH-SN (red) on days $\mathrm{L} 3$ (left) and PO (right), with the portion of the APR dendritic arbor ( green) that occupied the same volume of neuropil as the PH-SN superimposed. Images are oriented as in Figure 2 (anterior is up). The arbor of APR within sensory neuropil was less extensive on day $P 0$ than on day L3, providing fewer opportunities for synaptic contact (also see Fig. 4). B, Each panel shows a two-dimensional projection of the same reconstructed PH-SNs (red) that appear in $A$. The locations of putative output synapses onto APR are marked in yellow (the yellow dots indicate the location, not the size, of the putative synaptic contacts). Arrowheads indicate the site at which the PH-SN axons entered the neuropil. In this example, there were 43 putative synaptic contacts between the posterior PH-SN and APR on day L3 and four putative synaptic contacts on day $\mathrm{PO}$.

port the hypothesis that the massive reduction in the number of physical contacts between PH-SNs and APRs (Fig. $6 \mathrm{~A}$ ) plays a predominant role in the developmental reduction in EPSP amplitude. Fewer contacts should produce less total synaptic current, and hence a smaller EPSP. Relationships between the number of synaptic contacts and synaptic strength at monosynaptic connections have been amply demonstrated in other systems [crayfish (Nakagawa and Mulloney, 2001), mollusc (Glanzman et al., 1989), frog (Kuno et al., 1971; Grantyn et al., 1984), goldfish (Korn et al., 1981), and cat (Malenka and Nicoll, 1997)]. By this scenario, the predominant locus for the weakening of synapses from PH-SNs to APRs is postsynaptic, because of the steroid-induced regression of APR dendrites.

\section{Relationship to neural circuits for behavior}

The excitatory synapses from $\mathrm{PH}-\mathrm{SNs}$ to APRs constitute the direct pathway in the neural circuit for the larval proleg withdrawal reflex (Fig. 1C) and are the major source of synaptic drive to proleg retractor motoneurons during the reflex (Jacobs and Weeks, 1990; Streichert and Weeks, 1995). Hence, the steroid-induced weakening of these synapses has direct behavioral relevance in contributing to the weakening and loss of the proleg withdrawal reflex at the end of larval life. After pupation, some APRs are eliminated by programmed cell death, whereas the others undergo steroid-induced dendritic regrowth, receive new synaptic inputs, and are respecified for new functional roles (Weeks and Ernst-Utzschneider, 1989; Sandstrom and Weeks, 1998; Lubischer et al., 1999). Dendritic regression and synaptic loss extricates APRs from outmoded larval circuits and prepares the motoneurons to be incorporated into new circuits for the next life stages. The PH-SNs are believed to die after pupation (Streichert and Weeks, 1995) and so are not expected to undergo regression.

Our findings represent the first demonstration in a single system that a steroid-induced change in dendritic ar-

mal dendritic regression (Streichert and Weeks, 1995). The EPSP amplitude decreases between days L3 and P0 at these heterochronic mosaic synapses, suggesting that genomic actions of $20 \mathrm{E}$ on $\mathrm{PH}-\mathrm{SNs}$ are not required for synaptic weakening. On the postsynaptic side, the possibility that $20 \mathrm{E}$ affects the electrophysiological properties of APR, such as the number or density of ACh receptors, can be investigated in cell culture (Melville et al., 2003; and our unpublished observations).

Although such factors could contribute to synaptic weakening, the existing anatomical and electrophysiological data sup- chitecture is associated with a change in the strength of monosynaptic connections and a change in behavior. Steroid hormones have pervasive effects on dendritic architecture and behavior (see Introduction), but the demonstration of specific links between hormones, synapses, and behavior is difficult. Many fundamental issues regarding steroid effects on neurons and behavior can be readily addressed in the experimentally accessible insect nervous system (for review, see Fahrbach and Weeks, 2002). Application of this level of analysis to the verte- 
brate nervous system should reveal intriguing insights into the regulation of sexually differentiated and other behaviors by steroid hormones.

\section{References}

Bell RA, Joachim FA (1976) Techniques for rearing laboratory colonies of tobacco hornworms and pink bollworms. Ann Entomol Soc Am 69:365-373.

Blagburn JM, Beadle DJ, Sattelle DB (1985) Development of synapses between identified sensory neurones and giant interneurones in the cockroach Periplaneta americana. J Embryol Exp Morphol 86:227-246.

Bollenbacher WE, Smith SL, Goodman W, Gilbert WI (1981) Ecdysteroid titer during the larval-pupal-adult development of the tobacco hornworm, Manduca sexta. Gen Comp Endocrinol 44:302-306.

Buhl EH, Han ZS, Lorinczi Z, Stezhka VV, Karnup SV, Somogyi P (1994) Physiological properties of anatomically identified axo-axonic cells in the rat hippocampus. J Neurophysiol 71:1289-1307.

Davis GW, Goodman CS (1998) Genetic analysis of synaptic development and plasticity: homeostatic regulation of synaptic efficacy. Curr Opin Neurobiol 8:149-156.

DeRiemer SA, Macagno ER (1981) Light microscopic analysis of contacts between pairs of identified leech neurons with combined use of horseradish peroxidase and Lucifer yellow. J Neurosci 1:650-657.

Duch C, Levine RB (2000) Remodeling of membrane properties and dendritic architecture accompanies the postembryonic conversion of a slow into a fast motoneuron. J Neurosci 20:6950-6961.

Edwards FA (1995) Anatomy and electrophysiology of fast central synapses lead to a structural model for long-term potentiation. Physiol Rev 75:759-787.

Fahrbach SE, Weeks JC (2002) Hormonal regulation of neural and behavioral plasticity in insects. In: Hormones, brain, and behavior, Vol 3 (Pfaff D, Arnold A, Etgen A, Fahrbach S, Rubin R, eds), pp 331-358. San Diego: Academic.

García-Segura LM, Chowen JA, Parducz A, Naftolin F (1994) Gonadal hormones as promoters of structural synaptic plasticity: cellular mechanisms. Prog Neurobiol 44:279-307.

Glanzman DL, Kandel ER, Schacher S (1989) Identified target motor neuron regulates neurite outgrowth and synapse formation of Aplysia sensory neurons in vitro. Neuron 3:441-450.

Gonchar Y, Turney S, Price JL, Burkhalter A (2002) Axo-axonic synapses formed by somatostatin-expressing GABAergic neurons in rat and monkey visual cortex. J Comp Neurol 443:1-14.

Grantyn R, Shapovalov AI, Shiriaev BI (1984) Relation between structural and release parameters at the frog sensory-motor synapse. J Physiol (Lond) 349:459-474.

Granzow B, Friesen WO, Kristan WB (1985) Physiological and morphological analysis of synaptic transmission between leech motor neurons. J Neurosci 5:2035-2050.

Gray JR, Weeks JC (1999) Anatomical correlates of a steroid induced change in synaptic strength during development. Soc Neurosci Abstr 25:2174.

Jacobs GA, Weeks JC (1990) Postsynaptic changes at a sensory-to-motoneuron synapse contribute to the developmental loss of a reflex behavior during insect metamorphosis. J Neurosci 10:1341-1356.

Kent KS, Levine RB (1993) Dendritic reorganization of an identified neuron during metamorphosis of the moth Manduca sexta: the influence of interactions with the periphery. J Neurobiol 24:1-22.

Korn H, Triller A, Mallet A, Faber DS (1981) Fluctuating responses at a central synapse: $n$ of binomial fit predicts number of stained presynaptic boutons. Science 213:898-901.

Kuno M, Turkanis SA, Weakly JM (1971) Correlation between nerve terminal size and transmitter release at the neuromuscular junction of the frog. J Physiol (Lond) 213:545-556.

Kurz EM, Sengelaub DR, Arnold AP (1986) Androgens regulate the dendritic length of mammalian motoneurons in adulthood. Science 232:395-398.

Lamotte d'Incamps B, Destombes J, Thiesson D, Hellio R, Lasserre X, Kouchtir-Devanne N, Jami L, Zytnicki D (1998) Indications for GABAimmunoreactive axo-axonic contacts on the intraspinal arborization of a Ib fiber in cat: a confocal microscope study. J Neurosci 18:10030-10036.

Levine RB, Weeks JC (1989) Reorganization of neural circuits and behavior during insect metamorphosis. In: Perspectives in neural circuits and behavior (Carew TJ, Kelley D, eds), pp 195-228. New York: Liss.

Lubischer JL, Verhegge LD, Weeks JC (1999) Respecified larval proleg and body wall muscles circulate hemolymph in developing wings of Manduca sexta pupae. J Exp Biol 202:787-796.

Magoski NS, Bulloch AGM (1997) Localization, physiology, and modulation of a molluskan dopaminergic synapse. J Neurobiol 33:247-264.

Malenka RC, Nicoll RA (1997) Silent synapses speak up. Neuron 19:473-476.

Matsumoto A, Micevych PE, Arnold AP (1988) Androgen regulates synaptic input to motoneurons of the adult rat spinal cord. J Neurosci 8:4168-4176.

Melville JM, Hoffman KL, Jarrard HE, Weeks JC (2003) Cell culture of mechanoreceptor neurons innervating proleg hairs in Manduca sexta larvae, and co-culture with target motoneurons. Cell Tissue Res 311:117-130.

McEwen BS (2000) Effects of adverse experiences for brain structure and function. Biol Psychiatry 48:721-731.

Muller KJ, McMahan UJ (1976) The shapes of sensory and motor neurones and the distribution of their synapses in ganglia of the leech: a study using intracellular injection of horseradish peroxidase. Proc R Soc Lond B Biol Sci 197:481-499.

Nakagawa H, Mulloney B (2001) Local specification of relative strengths of synapses between different abdominal stretch-receptor axons and their common target neurons. J Neurosci 21:1645-1655.

Nijhout HF, Williams CM (1974) Control of moulting and metamorphosis in the tobacco hornworm, Manduca sexta (L.): growth of the last-instar larva and the decision to pupate. J Exp Biol 61:481-491.

Peters BH, Altman JS, Tyrer NM (1985) Synaptic connections between the hindwing stretch receptor and flight motor neurones in the locust revealed by double cobalt labelling for electron microscopy. J Comp Neurol 233:269-284

Peterson BA, Weeks JC (1988) Somatotopic mapping of sensory neurons innervating mechanosensory hairs on the larval prolegs of Manduca sexta. J Comp Neurol 275:128-144.

Pilowsky PM, deCastro D, Llewellen-Smith I, Lipski J, Voss MD (1990) Serotonin immunoreactive boutons make synapses with feline phrenic motoneurons. J Neurosci 10:1091-1098.

Riddiford LM, Truman JW (1994) Hormone receptors and the orchestration of development during insect metamorphosis. In: Perspectives in comparative endocrinology, pp 389-394. National Research Council of Canada.

Sandstrom DJ, Weeks JC (1996) Novel dual innervation of a larval proleg muscle by two similar motoneurons in the tobacco hornworm Manduca sexta. J Exp Biol 199:775-791.

Sandstrom DJ, Weeks JC (1998) Segment-specific retention of a larval neuromuscular system and its role in a new, rhythmic, pupal motor pattern in Manduca sexta. J Comp Physiol [A] 183:283-302.

Sorra KE, Harris KM (1993) Occurrence and three-dimensional structure of multiple synapses between individual radiatum axons and their target pyramidal cells in hippocampal area CA1. J Neurosci 13:3736-3748.

Stewart BA, Schuster CM, Goodman CS, Atwood HL (1996) Homeostasis of synaptic transmission in Drosophila with genetically altered nerve terminal morphology. J Neurosci 16:3877-3886.

Streichert LC, Weeks JC (1995) Decreased monosynaptic sensory input to an identified motoneuron is associated with steroid-mediated dendritic regression during metamorphosis in Manduca sexta. J Neurosci 15:1484-1495.

Sun XJ, Tolbert LP, Hildebrand JG (1997) Synaptic organization of the uniglomerular projection neurons of the antennal lobe of the moth Manduca sexta: a laser scanning confocal and electron microscopic study. J Comp Physiol [A] 379:2-20.

Trimmer BA, Weeks JC (1989) Effects of nicotinic and muscarinic agents on an identified motoneurone and its direct afferent inputs in larval Manduca sexta. J Exp Biol 144:303-337.

Tse FW, Marin L, Jahromi SS, Atwood HL (1991) Variation in terminal morphology and presynaptic inhibition at crustacean neuromuscular junctions. J Comp Neurol 304:135-146.

Tsujimoto T, Umemiya M, Kuno M (1990) Terminal sprouting is not responsible for enhanced transmitter release at disused neuromuscular junctions of the rat. J Neurosci 10:2059-2065.

Walmsley B, Wieniawa-Narkiewwicz E, Nichol MJ (1987) Ultrastructural evidence related to presynaptic inhibition of primary muscle afferents in Clarke's column of the cat. J Neurosci 7:236-243.

Watson AHD, Burrows M (1982) The ultrastructure of identified locust motor neurones and their synaptic relationships. J Comp Neurol 205:383-397.

Watson AHD, Burrows M (1985) The distribution of synapses on the two 
fields of neurites of spiking local interneurones in the locust. J Comp Neurol 240:219-232.

Weeks JC (1987) Time course of hormonal independence for developmental events in neurons and other cell types during insect metamorphosis. Dev Biol 124:163-176.

Weeks JC, Ernst-Utzschneider K (1989) Respecification of larval proleg motoneurons during metamorphosis of the tobacco hornworm, Manduca sexta: segmental dependence and hormonal regulation. J Neurobiol 20:569-592.

Weeks JC, Jacobs GA (1987) A reflex behavior mediated by monosynaptic connections between hair afferents and motoneurons in the larval tobacco hornworm, Manduca sexta. J Comp Physiol [A] 160:315-329.

Weeks JC, McEwen BS (1997) Modulation of neural circuits by steroid hormones in rodent and insect model systems. In: Neurons, networks, and motor behavior (Stein PSG, Grillner S, Selverston AI, eds), pp 195-207. Cambridge, MA: MIT.

Weeks JC, Truman JW (1985) Independent steroid control of the fates of motoneurons and their muscles during insect metamorphosis. J Neurosci 5:2290-2300.
Weeks JC, Truman JW (1986) Hormonally mediated reprogramming of muscles and motoneurones during the larval-pupal transformation of the tobacco hornworm, Manduca sexta. J Exp Biol 125:1-13.

Weeks JC, Roberts WM, Trimble DL (1992) Hormonal regulation and segmental specificity of motoneuron phenotype during metamorphosis in Manduca sexta. Dev Biol 149:185-196.

Weeks JC, Jacobs GA, Pierce JT, Sandstrom DJ, Streichert LC, Trimmer, Wiel DE, Wood ER (1997) Neural mechanisms of behavioral plasticity: metamorphosis and learning in Manduca sexta. Brain Behav Evol 50 [Suppl 1]:69-80.

Wiel D, Weeks JC (1996) Habituation and dishabituation of the proleg withdrawal reflex in larvae of the hawk moth, Manduca sexta. Behav Neurosci 5:1133-1147.

Woolley CS (1998) Estrogen-mediated structural and functional synaptic plasticity in the female rat hippocampus. Horm Behav 34:140-148.

Yankova M, Hart SA, Woolley CS (2001) Estrogen increases synaptic connectivity between single presynaptic inputs and multiple postsynaptic CA1 pyramidal cells: a serial electron-microscopic study. Proc Natl Acad Sci USA 98:3525-3230. 